INRA Prod. Anim.,

2014, 27 (5), 347-358

\title{
Objectivation de la notion de grain de viande et perspectives d'utilisation pour évaluer la tendreté des viandes de bovins Charolais
}

\author{
M.-P. ELLIES-OURY ${ }^{1,5,6}$, Y. DURAND ${ }^{2,3}$, A.-E. DELAVIGNE ${ }^{4}$, B. PICARD ${ }^{5,6}$, D. MICOL ${ }^{5,6}$, R. DUMONT \\ ${ }^{1}$ Bordeaux Sciences Agro, 1 cours du Général de Gaulle, CS 40201, F-33175 Gradignan, France \\ 2 Institut Charolais, N79 Route Centre Europe Atlantique, F-71120 Charolles, France \\ ${ }^{3}$ Chambre d'Agriculture de Saône et Loire, 59 rue du 9 mars 1962, F-71000 Mâcon, France \\ ${ }^{4}$ MNHN-CNRS Eco Anthropologie et Ethnologie UMR7206, Ilôt Poliveau, 57 rue Cuvier, F-75231 Paris, France \\ 5 INRA, UMR1213 Herbivores, F-63122 Saint-Genès-Champanelle, France \\ ${ }^{6}$ Clermont Université, VetAgro Sup, UMR1213 Herbivores, BP 10448, F-63000, Clermont-Ferrand, France \\ ${ }^{7}$ AgroSup Dijon, BP 87999, F-21079 Dijon, France \\ Courriel : marie-pierre.ellies@agro-bordeaux.fr
}

Le grain de viande est une notion utilisée dans certains cahiers des charges en viande Charolaise pour classer les carcasses et les orienter sur le marché selon leur qualité. Mais cette notion doit d'abord être objectivée et formalisée dans une grille de jugement des carcasses et des animaux sur pied pour juger de sa pertinence à évaluer précocement la tendreté des viandes.

En zone Charolaise, des professionnels jugent couramment du « potentiel viande » des animaux. Pour cela, ils évaluent la précocité physiologique (c'est-à-dire l'aptitude à s'engraisser plus ou moins rapidement), ce qui est important pour choisir la durée du cycle de production et le meilleur âge à l'abattage. Ils apprécient également la conformation de l'animal, le rendement en viande de la carcasse et le « grain de viande ». Cette dernière notion empirique s'acquiert avec l'expérience et dans la durée. Elle fait partie de la culture acquise et partagée par les professionnels de la filière bovine Charolaise. Selon eux, " la finesse du grain de viande », serait à l'origine d'une part importante de la tendreté potentielle de la viande, la quantification de ce critère étant basée sur la notation d'un ensemble d'indicateurs empiriques identifiés sur l'animal et/ou sur sa carcasse. Les indicateurs utilisés regroupent plusieurs informations visuelles et tactiles comme par exemple la finesse des os et du cuir ou encore la sensation au toucher de différents muscles, mais leur appréciation semble propre à chaque opérateur. Ces pratiques permettent d'orienter les carcasses vers des circuits commerciaux ad hoc afin de répondre aux attentes des clients.

S'il apparaît indispensable de s'appuyer sur l'expérience, le professionnalisme et le savoir-faire des opérateurs de la filière pour repérer les données subjectives utilisées empiriquement pour définir les prédicteurs de la qualité des viandes, l'efficacité de ces méthodes reste malgré tout à démontrer et les critères de jugement sur lesquels elles reposent doivent être objectivés.

Les recherches sur les savoir-faire en matière d'évaluation des animaux et de la qualité organoleptique de leur viande restent pour le moment assez limitées et sont vraisemblablement risquées. Il faut toutefois citer un essai cherchant à évaluer la fiabilité de l'expertise professionnelle pour distinguer les types « précoce» et " tardif » en races Charolaise et Limousine (Grenet et al 2000). Ce travail, qui n'a pas pu mettre en évidence de différences de performances à l'abattage et de qualité de viande entre les deux précocités annoncées à l'expertise, conclut à l'absence de fiabilité de l'expertise de départ ou au mauvais choix des indicateurs.

Une tentative d'étude du grain de la viande a été faite en 1997 (Jabet et Turin 1997). Il s'agissait de mettre au point une grille de notation capable de reproduire de façon fiable le jugement des professionnels. Les observations étaient faites après abattage sur un seul muscle prélevé au niveau de la sixième côte.
L'enquête préalable n'a pas permis de définir des indicateurs communs d'observation du grain de viande dans le cadre particulier de ce travail (évaluation d'une tranche de longissimus thoracis). De même, les appréciations de différents morceaux faites par quatre professionnels se sont révélées peu reproductibles et peu répétables. Cette étude a cependant soulevé deux questions importantes :

- le « grain de viande » s'apprécie-t-il en vif ou sur la carcasse ou sur un morceau isolé ?

- ne serait-il pas utile d'aborder la question de manière plus générale en identifiant les savoir-faire pour la prédiction de la tendreté d'une viande plutôt que d'entrer par la notion de « grain de la viande »?

Ces tentatives d'exploration des savoirfaire méritent donc d'être développées C'est ce que nous avons entrepris dans une série d'études dont nous présentons ici la synthèse. Ainsi, nous avons cherché à :

- montrer par une approche ethnologique la réalité de la notion de « grain de viande » dans le champ des savoirfaire des professionnels de la viande en zone Charolaise ;

- formaliser l'évaluation du « grain » en établissant une grille reproductible et utilisable par un jury d'évaluation ; 
- établir les relations entre le " grain» (grain grossier à très fin) et les caractéristiques physico-chimiques musculaires ou la qualité sensorielle de la viande (en particulier la tendreté).

\section{1 / La notion de grain de viande : un savoir-faire em- pirique pour apprécier la qualité bouchère d'une bête et/ou d'une carcasse}

Dans un premier temps, une étude des savoir-faire des professionnels de la filière viande charolaise en matière d'évaluation des animaux et de la qualité organoleptique de leur viande a été commanditée dans le cadre de l'appel à projets Agriculture et Développement Durable de l'ANR (campagne 2005). Cette étude ethnologique a été réalisée par A-E. Delavigne (UMR Ressources des terroirs - cultures, usages et sociétés, CNRS).

Pour appréhender les savoir-faire des professionnels, la méthode la plus adaptée semblait être la méthode ethnologique, susceptible d'apporter des matériaux de première main permettant de dresser un état des lieux de la question dans toute sa complexité. L'essentiel de la collecte de données a été basée sur des entretiens longs, non directifs et répétés. Ils ont eu lieu dans la mesure du possible en situation, c'est-à-dire mettant en œuvre des observations directes en présence des animaux ce qui permettait d'expliciter certains des savoir-faire empiriques.

Les personnes à enquêter étaient les différentes catégories de professionnels de la filière bovine charolaise ayant à faire à l'animal en vif et en carcasse. Pour cette étude ethnologique cinquante professionnels de quatre terrains successifs (Saône-et-Loire, Allier, Nièvre et Anjou) ont été interviewés (Delavigne $2008 \mathrm{a}$ et b). Cet échantillon de professionnels a été considéré comme représentatif de la filière viande bovine charolaise. Il a été constitué grâce au réseau de connaissances et d'interconnaissances et comptait des professionnels ayant à faire à l'animal en vif (7 éleveursengraisseurs, 2 emboucheurs, 1 sélectionneur, 3 responsables de groupement et techniciens) ou en carcasse ( 6 chevillards ou négociants en bestiaux, 1 transformateur, 30 bouchers abatteurs).

Pour mettre au jour les représentations et pratiques de ces professionnels et contourner la difficulté de transcrire un jugement subjectif réalisé par les opérateurs de la filière, le questionnement n'a pas été directement sur le " grain de viande $»^{1}$. L'idée était de tenter de saisir cette notion au travers de catégories constitutives de la notion de « qualité ».

L'analyse a porté sur la définition de la « qualité » recherchée par les personnes rencontrées, sa catégorisation à partir des entretiens permettant la mise en évidence des principaux indicateurs d'évaluation de la qualité des carcasses et des viandes et notamment de la notion de tendreté et de « grain de la viande ».

Il ressort de cette étude que le savoirfaire d'appréciation du grain de viande est empirique et s'acquiert par transmission, mais aussi avec l'expérience et s'inscrit dans la durée.

Il existe une grande convergence des indicateurs cités et un relatif consensus au sein de la profession autour de ce terme, notamment sur le fait que cette notion est positive et sert à qualifier une " bonne bête », une « bonne viande», un «bon morceau ». Le grain de viande est cependant seulement un critère parmi un ensemble de critères pour aider au choix d'une bête ou d'une carcasse en fonction des besoins du boucher, des attentes du client, etc. Pour les professionnels, l'indication fournie par le grain de viande donne une indication de la valorisation possible de la carcasse et une information sur la tendreté probable de la viande.

Lors de l'achat en vif, les professionnels prêtent attention à un certain nombre de « repères pour la viande » qui leur permettent de mettre toutes les chances de leur côté dans le choix d'un animal : par exemple, ils préfèrent un animal avec un cuir fin et élastique, un poil frais, des cornes en bascule, un museau fin et allongé, etc. Pour juger du grain sur la carcasse ou lors de la coupe de l'arrière tranché à 8 côtes (ART8), les professionnels utilisent une combinaison de descripteurs faisant intervenir différentes dimensions sensorielles ( il faut avoir l'œil et le doigt »). Ainsi, pour apprécier le grain de viande sur la carcasse ou lors de la découpe de détail, les professionnels combinent une association du toucher et de la vue et prêtent également attention au $"$ relief $»$. La vue et le toucher sont ainsi des sens difficilement séparables et qui se complètent pour donner une indication sur la qualité supposée de la viande. Ces différentes approches semblent bien complémentaires et indispensables à une évaluation précise du grain de viande sur la carcasse alors qu'elles étaient considérées comme divergentes par Jabet et Turin (1997).
Les professionnels évaluent ainsi à la fois des « repères pour la viande » lorsque l'animal est sur pied et que l'achat des animaux se fait en vif, mais également sur la demi-carcasse et/ou après la coupe en quartiers, le professionnel jugeant alors « au doigt et à l'ail ».

Suite à cette étude, des points restaient à préciser notamment sur les observations à effectuer pour juger le grain de viande sur animal vivant et sur carcasse, la définition des termes et le lien objectif entre grain de viande et tendreté. Aussi, les différents « repères pour la viande » ont-ils été décrits et intégrés dans deux grilles parallèles : sur animal vivant et sur carcasse (Oury et al 2008, 2010a, 2010b, Ellies-Oury et al 2011).

\section{2 / Formalisation de la notion de grain de viande dans deux grilles de notation reproduc- tibles et utilisables sur le terrain}

\section{1 / Construction d'une grille d'évaluation du grain de viande sur la carcasse}

La première étape a permis d'identifier un nombre conséquent d'indicateurs susceptibles d'être reliés au grain de viande sur la carcasse et la viande (caractéristiques propres de l'animal, indicateurs sur la carcasse, à la coupe et à la découpe).

Suite à l'analyse détaillée des conclusions de l'étude ethnologique, un boucher et un chevillard utilisant couramment la notion de grain de viande, ont été sollicités pour sélectionner et hiérarchiser les différents indicateurs à évaluer sur les carcasses. Issus du berceau de race Charolais et couramment sollicités pour le jugement des carcasses de l'AOC Bœuf de Charolles, ces deux experts ont été considérés comme les plus à même de co-construire la grille de notation du grain de viande. Pour chaque indicateur indiqué comme pertinent, la méthode d'observation a été décrite le plus précisément possible. Les modalités extrêmes de chaque indicateur ont été précisées et illustrées. Une grille provisoire a été élaborée dans laquelle les modalités de chaque indicateur ont été retranscrites dans des cases à cocher selon les indications des deux professionnels. Ceux-ci ont, en outre, identifié des carcasses qui correspondaient aux notes extrêmes des indicateurs les plus délicats à noter afin d'illustrer la grille avec des photos. Certains maniements et zones d'observation

${ }^{1}$ Les guillemets ont été utilisés pour retranscrire le vocabulaire utilisé par les acteurs de la filière lors des entretiens ethnologiques. 
Tableau 1. Indicateurs relevés sur la carcasse entière et après découpe, et notes attribuées selon les modalités identifiées avec les experts (d'après Oury et al 2010a et b, Ellies-Oury et al 2011).

\begin{tabular}{|c|c|c|}
\hline & Note de $1^{*}$ & Note de $5^{*}$ \\
\hline \multicolumn{3}{|l|}{ Appréciation sur la carcasse entière } \\
\hline Appréciation de la carcasse & $\begin{array}{l}\text { Carcasse trop grande, beaucoup } \\
\text { de poitrine, creuse, cabarde }\end{array}$ & $\begin{array}{l}\text { Carcasse bien conformée, } \\
\text { courte, rondelette }\end{array}$ \\
\hline Equilibre avant-arrière & $\begin{array}{l}\text { Carcasse mal équilibrée, } \\
\text { trop d'avants }\end{array}$ & $\begin{array}{l}\text { Carcasse bien équilibrée, } \\
\text { peu d'avants }\end{array}$ \\
\hline Conformation de la cuisse & Cuisse manquant de rondeur & $\begin{array}{l}\text { Cuisse bien ronde } \\
\text { et régulière }\end{array}$ \\
\hline $\begin{array}{l}\text { Appréciation du développement osseux } \\
\text { au niveau de la crosse }\end{array}$ & Crosse grossière & Crosse fine \\
\hline $\begin{array}{l}\text { Appréciation de l'état d'engraissement } \\
\text { de la carcasse }\end{array}$ & Carcasse maigre ou très grasse & Carcasse couverte \\
\hline Evaluation du suintement sous la carcasse & Suintement absent ou excessif & Léger suintement \\
\hline Sensation au toucher de la hampe & Fibres dures et sèches & $\begin{array}{l}\text { Fibres écrasantes } \\
\text { et souples }\end{array}$ \\
\hline Sensation au toucher du filet de côte & Fibres dures et sèches & $\begin{array}{l}\text { Fibres écrasantes } \\
\text { et souples }\end{array}$ \\
\hline \multicolumn{3}{|l|}{ Appréciation après découpe } \\
\hline Epaisseur relative du muscle & $\begin{array}{l}\text { Pas d'épaisseur de muscle, forte } \\
\text { épaisseur de gras }\end{array}$ & $\begin{array}{l}\text { Très bonne épaisseur de } \\
\text { muscle, très peu de gras }\end{array}$ \\
\hline $\begin{array}{l}\text { Présence de gras intermusculaire au niveau } \\
\text { de la } 5^{\text {eme }} \text { côte }\end{array}$ & Teneur très limitée de gras & $\begin{array}{l}\text { Teneur très importante de } \\
\text { gras }\end{array}$ \\
\hline $\begin{array}{l}\text { Observation de la noix de côte au niveau } \\
\text { de la coupe }\end{array}$ & La coupe ne perle pas & La coupe perle \\
\hline Observation des nerfs sur la noix de côte & Beaucoup de nerfs visibles & $\begin{array}{l}\text { Absence totale de nerfs } \\
\text { visibles }\end{array}$ \\
\hline Observation du persillé sur la noix de côte & $\begin{array}{l}\text { Persillé très visible, zones de gras } \\
\text { très épaisses }\end{array}$ & $\begin{array}{l}\text { Absence de persillé, persillé } \\
\text { invisible }\end{array}$ \\
\hline Observation des fibres de la noix de côte & Fibres très grosses, très visibles & $\begin{array}{l}\text { Fibres très fines, très peu } \\
\text { visibles }\end{array}$ \\
\hline $\begin{array}{l}\text { Sensation au toucher de la noix de côte } \\
\text { et du rhomboïde thoracique }\end{array}$ & $\begin{array}{l}\text { Sensation de très rugueux, très } \\
\text { granuleux }\end{array}$ & $\begin{array}{l}\text { Sensation de lisse, doux, } \\
\text { sans aspérités }\end{array}$ \\
\hline Appréciation globale du grain de viande & Grain très grossier & Grain extrêmement fin \\
\hline
\end{tabular}

${ }^{*}$ Chacun des indicateurs est noté de 1 à 5 ; la note de 1 est la moins favorable pour le grain de viande, à linverse de la note de 5. 
ont également été photographiés afin de permettre une diffusion ultérieure de la grille. Les définitions des différents indicateurs (basées sur l'enquête ethnologique et le discours des deux professionnels) ont enfin été précisées dans une liste proposée en parallèle de la grille.

La grille a été construite en deux sousparties : une sur la carcasse entière, l'autre après séparation de l'ART8. L'appréciation sur la carcasse entière, réalisée au moins 24 heures post mortem, avant la séparation entre avants et arrières, comporte 8 indicateurs notés entre 1 et 5 (l'appréciation du développement osseux et de l'état d'engraissement, l'équilibre avant-arrière, la sensation au toucher de différents muscles...). Pour chaque indicateur, la note de 5 est attribuée à la modalité la plus favorable au grain de viande selon les professionnels. L'appréciation après découpe au niveau de la coupe du caparaçon est réalisée sur l'avant de la carcasse, si possible 48 heures post mortem. Elle comporte également 8 indicateurs (notamment relatifs à l'observation et au toucher de la noix de côte). Une note globale d'appréciation du grain de viande, synthèse de l'appréciation générale de la carcasse, a été ajoutée (tableau 1).

\section{2 / Validation et utilisation de la grille par les professionnels}

Cette grille provisoire a été affinée et validée en la soumettant à quatre experts. L'objectif était d'établir les liens entre les différents indicateurs de la grille, de les hiérarchiser (voire d'en éliminer certains) et de mettre en relation chacun des indicateurs avec la note globale d'appréciation du grain de viande. Afin de s'assurer de l'absence d'interaction des experts au cours des évaluations et assurer une indépendance de leur notation, les carcasses à évaluer étaient séparées les unes des autres par trois autres carcasses. Le même procédé a été employé pour l'ensemble des notations opérées par les experts.

Le test de la grille a porté sur 31 carcasses de vaches $(n=13)$ et génisses $(n=18)$ Charolaises, ayant un état d'engraissement moyen de 3 (25 carcasses sur 31$)$ et notées $\mathrm{U}$ ou R (13 et 11 carcasses respectivement) selon la conformation EUROP.

Parmi les quatre experts se trouvaient trois bouchers qui, dans leur activité quotidienne, ont l'habitude d'apprécier et de trier les carcasses dans les chambres froides de l'abattoir qui les approvisionne et un professionnel formé à l'évaluation du grain de viande par ces mêmes bouchers. L'objectif était d'évaluer la possibilité de former à l'évaluation du grain de viande, un professionnel sensibilisé à cette notion.
Pour l'ensemble des 17 indicateurs de la grille, le classement des carcasses pour les différents postes s'est révélé cohérent entre les quatre experts (coefficients de concordance de Kendall - CCK > 0,63 ; $\mathrm{P}<0,0001)$. Chacun des indicateurs étant évalué de la même façon par les quatre experts, la grille peut être considérée comme reproductible. Il n'est en revanche pas possible d'évaluer la répétabilité dans la mesure où les experts sont capables de reconnaître les carcasses qu'ils ont déjà évaluées. Il est donc impossible de leur présenter deux fois la même carcasse. Le classement des carcasses s'est également révélé concordant pour chacun des 17 indicateurs entre chacun des bouchers et le professionnel formé $(\mathrm{CCK}>0,60$; $\mathrm{P}<$ $0,0001)$. Ces résultats montrent donc qu'il est possible de former à l'évaluation du grain de viande, un professionnel sensibilisé à cette notion. En conclusion, il existe bien un consensus parmi les professionnels sur cette notion de grain de viande, qui s'avère être un savoir-faire transmissible.

Tous indicateurs confondus, les experts notent les carcasses avec 0,5 point d'écart dans $71 \%$ des cas et avec 1,5 points d'écart au maximum (dans $6 \%$ des cas). Lorsque l'on ne conserve que les notes attribuées par les trois bouchers, il n'y a pas d'écart de notation dans $56 \%$ des cas, et au maximum un écart de 0,5 point (dans $44 \%$ des cas). L'étagement des notations entre experts et donc tout à fait convenable et cohérent avec les résultats obtenus dans les tests réalisés pour le jugement selon la grille EUROP (écarts de 3 à 5 tiers de notes).

Jabet et Turin (1997) ont précédemment tenté de formaliser cette notion de grain de viande dans une grille de notation sans pour autant y parvenir. Cette différence de résultats peut s'expliquer par des différences dans le protocole employé. Ainsi, Jabet et Turin (1997) avaient sollicité des professionnels provenant de différents abattoirs et travaillant sur plusieurs races bovines. De plus ces auteurs ont établi la grille à partir de la seule observation d'une tranche de longissimus thoracis alors que dans le présent travail la notation du grain de viande a été réalisée directement sur la carcasse entière et sur l'ART8, sur une seule race et avec des experts familiers de cette race. Aussi faudra-t-il envisager à l'avenir de tester la pertinence et la possibilité d'étendre une telle grille d'évaluation du grain de viande à d'autres races que la race Charolaise

\section{3 / Relation entre le grain de vian- de et les différents indicateurs de la composition corporelle des animaux}

Parmi les indicateurs d'évaluation sur la demi-carcasse, les postes relatifs au développement des animaux (l'appréciation de la carcasse, l'équilibre avantarrière et la conformation de la cuisse) donnent une notation très concordante avec celle du grain de viande $(0,85<$ CCK $<0,89 ; 0,001<\mathrm{P}<0,01$; figure 1$)$ : les notes moyennes attribuées à ces postes évoluent en effet régulièrement en fonction du grain de viande (Oury et al 2010a et b, Ellies-Oury et al 2011).

Le fait que ces postes soient pris en compte également pour l'attribution de la note de conformation EUROP engage à chercher à établir le lien entre la conformation et la notation du grain de viande. La moyenne des notes de grain de viande diminue avec les classes de conformation (figure 2). Néanmoins, il est intéressant de constater qu'une carcasse peut avoir un bon grain (note de 3,8 sur 5) tout en étant conformée R+. A l'opposé, une carcasse conformée E peut avoir un grain peu fin (note de 3 sur 5). Aussi, si la conformation est un facteur favorable, il demeure une incertitude quand à la note de grain de viande pour une conformation donnée.

D'autres indicateurs peuvent être liés au grain de viande, notamment l'évaluation du développement osseux ou la sensation au toucher de la hampe et de la chaînette. Ces trois indicateurs ne présentent pas une évolution régulière en fonction du grain de viande, mais sont néanmoins très pertinents pour sélectionner les animaux exceptionnels dont la note de grain de viande est de 4,5 ou 5 (figure 1).

L'ensemble des huit postes évalués après découpe est très concordant avec l'appréciation globale du grain de viande $(0,93<\mathrm{CCK}<0,95 ; \mathrm{P}<0,001)$. Le classement des carcasses selon les postes d'évaluation du développement des tissus adipeux (épaisseur relative musclegras, absence de gras intermusculaire au niveau de la $5^{\text {èe }}$ côte, absence de persillé sur la noix de côte) est concordant avec celui du grain de viande (figure 3). Il faut donc bien noter que l'augmentation de la note globale de grain de viande va de pair avec la réduction du tissu adipeux. Pour un grain de viande fin, il faut favoriser :

- une augmentation de l'épaisseur relative muscle/gras ;

- une moindre teneur en gras intermusculaire au niveau de la coupe ;

- un persillé bien réparti et peu visible, voire totalement invisible.

Ces conclusions relatives aux teneurs et à la répartition optimale des tissus adipeux nous ont conduits à envisager le lien entre note d'état et grain de viande. Cependant, $80 \%$ des carcasses étant notées $3 / 5$ en état d'engraissement, il a 
Figure 1. Note moyenne de différents indicateurs observés sur la carcasse selon la note de grain de viande de la carcasse (résultats obtenus sur 31 carcasses de femelles Charolaises) (d'après Oury et al 2010a et b. Ellies-Oury et al 2011).

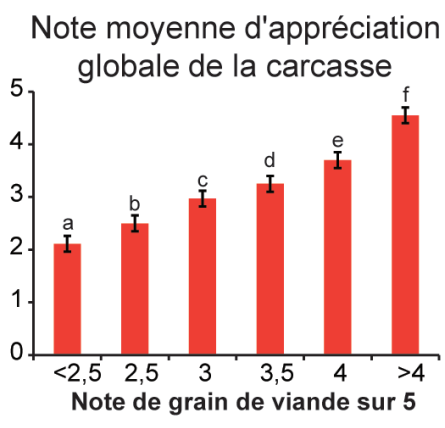

Note moyenne

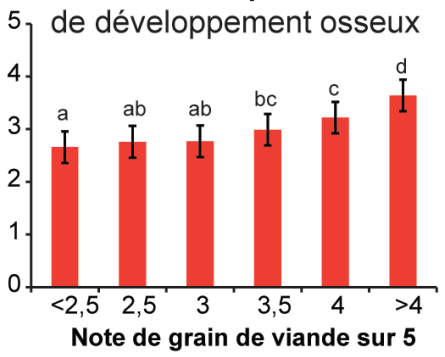

Note moyenne au toucher de la noix de côte

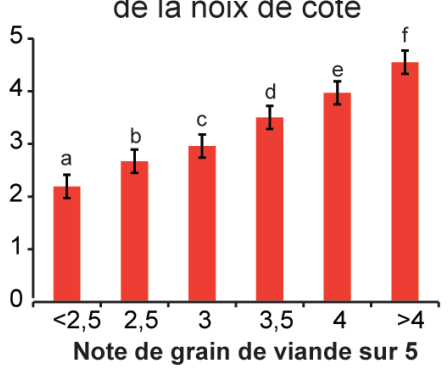

Note moyenne d'équilibre

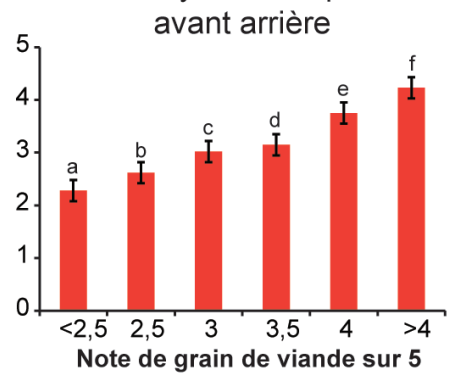

Note moyenne

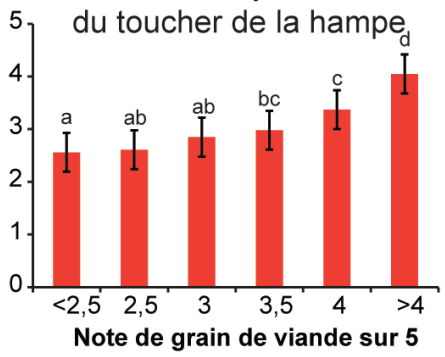

Note moyenne au toucher du rhomboïde thoracique

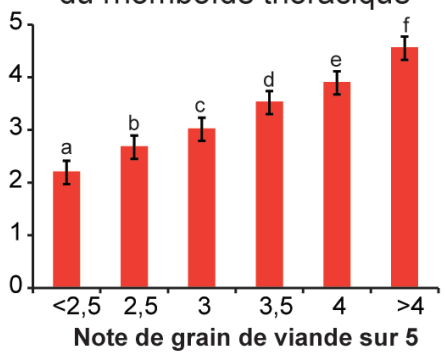

Note moyenne de conformation

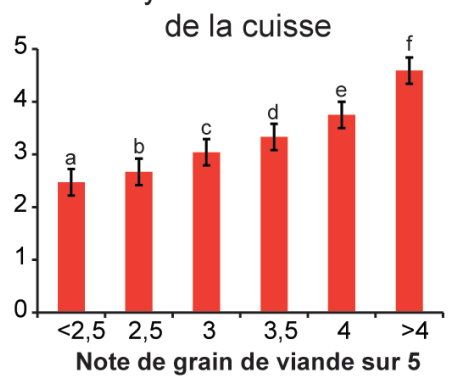

Note moyenne de toucher

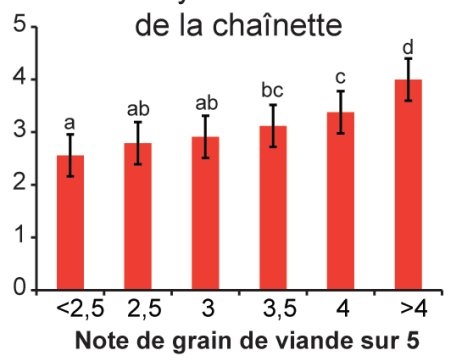

Une note de 5 pour chacun de ces descripteurs implique : une carcasse bien conformée, courte et rondelette ; bien équilibrée avec peu d'avants ; une cuisse bien ronde et régulière ; une crosse fine ; une sensation de fibres écrasantes et souples au toucher de la hampe et de la chaînette ; une sensation de lisse, doux, sans aspérité au toucher de la noix de côte et du rhomboïde thoracique.

Des lettres différentes témoignent d'une différence significative $(P<0,05)$.

Figure 2. Notes moyennes ( $\mathbf{\Delta}$ ), minimales et maximales de grain de viande de carcasses de femelles Charolaises selon les notes EUROP de a) conformation et b) d'état d'engraissement (résultats obtenus sur 31 carcasses de femelles Charolaises) (d'après Oury et al 2010a et b, Ellies-Oury et al 2011).

Notes moyennes, maximales et minimales

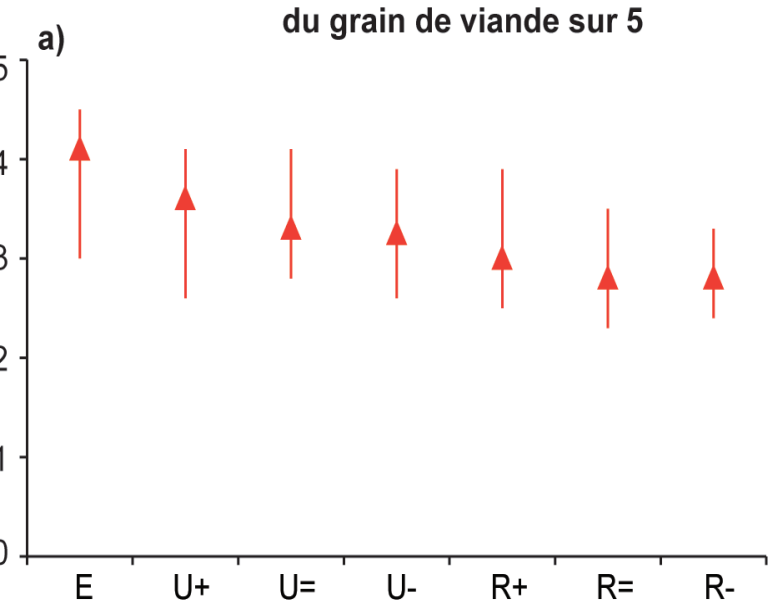

b)

Notes d'état moyennes sur 5

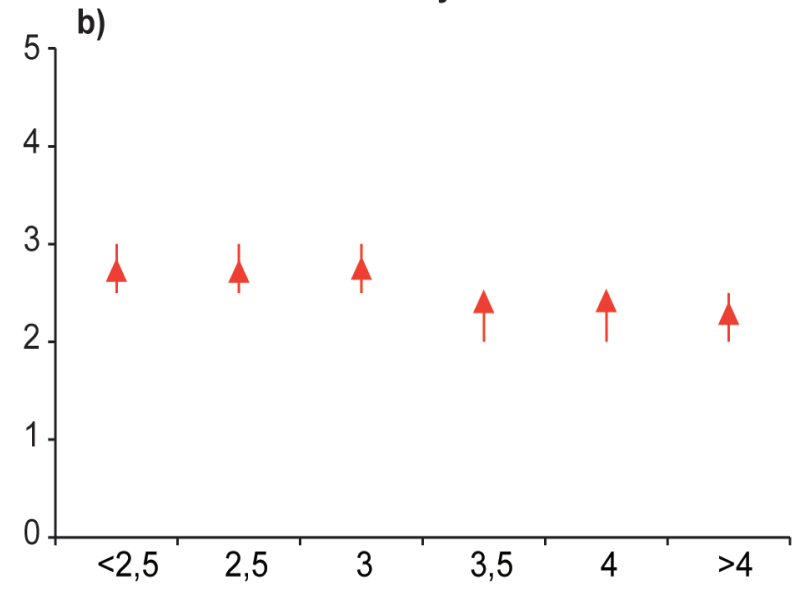

Conformation EUROP 
Figure 3. Notes moyennes (A), minimales et maximales des indicateurs relatifs au développement du tissu adipeux selon la note globale de grain de viande (résultats obtenus sur 31 carcasses de femelles Charolaises) (Oury et al 2010a et b, Ellies-Oury et al 2011).

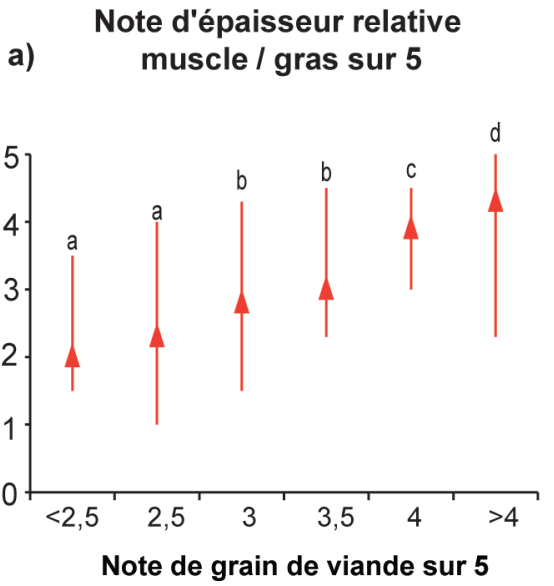

Note d'absence de gras
b) intermusculaire sur 5

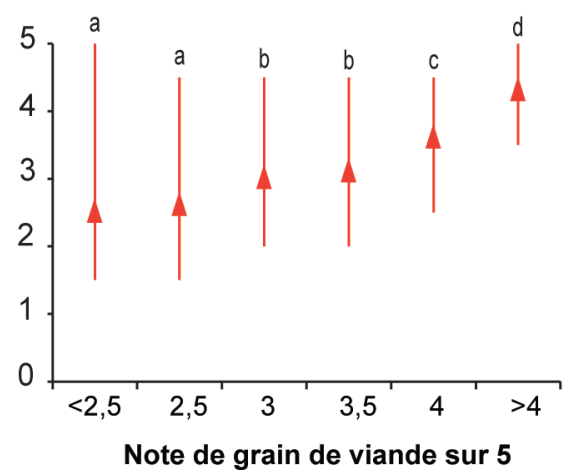

c)

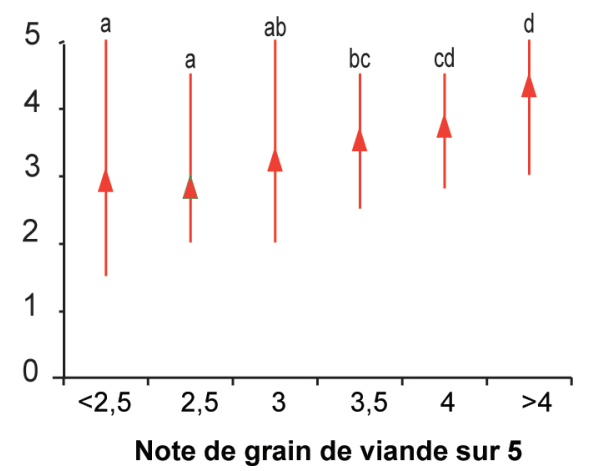

Une note de 5 pour chacun de ces trois descripteurs implique : a) une très bonne épaisseur de muscle avec très peu de gras ; b) une teneur très limitée de gras intermusculaire au niveau de la côte ; c) une absence de persillé ou un persillé non visible au niveau de la noix de côte. Des lettres différentes témoignent d'une différence significative $(P<0,05)$.

été impossible d'établir le lien entre les notes d'état d'engraissement et le grain de viande (figure 2 ).

Les deux indicateurs les plus efficaces pour évaluer le grain de viande sont les indicateurs relatifs au toucher de la noix de côte (longissimus thoracis) et du rhomboïde thoracique avec une évolution synchrone des notes moyennes pour chacun de ces deux postes en fonction de la note de grain de viande (figure 1) : un grain de viande extrêmement fin va de pair avec un toucher lisse, doux et sans aspérités de ces deux muscles de la côte.

De l'évaluation de la carcasse il ressort donc que pour obtenir une viande à grain extrêmement fin, il est préférable d'avoir une carcasse bien conformée, bien équilibrée, ayant une cuisse bien ronde ainsi qu'une hampe et une châ̂nette écrasantes et souples. Lorsqu'il est possible d'avoir accès à l'ART8, les indicateurs d'évaluation du grain de viande sont beaucoup plus pertinents. Dans ce cas, les chances d'avoir un grain fin sont améliorées en prenant une carcasse à forte épaisseur de muscle, ayant une teneur limitée de gras intermusculaire, une noix de côte dont la coupe perle, sans nerfs, sans persillé, avec des fibres fines et un toucher lisse, doux et soyeux.

\section{4 / Construction d'une grille d'évaluation du grain de viande sur l'animal vif}

Dans un second temps, le travail a consisté à expliciter certains des « repères sur la viande » identifiables en vif afin d'élaborer une grille de notation utilisable avant abattage (Oury et al 2010b). Pour cela, un chevillard et trois bouchers ayant l'habitude d'acheter en vif en utilisant la notion de grain de viande ont été sollicités afin d'identifier les indicateurs clefs de notation ainsi que les maniements nécessaires à leur notation, les modalités extrêmes et la modalité la plus favorable. Pour ce faire, ils ont été interrogés sur les principaux repères indentifiables sur l'animal vif permettant de renseigner sur la qualité ultérieure de la viande.

Une première ébauche de grille, complétée par une planche de photos, a été proposée puis validée par les experts avant d'être mise à l'épreuve selon le même procédé que pour la grille sur la carcasse. Pour cela, 60 femelles Charolaises ont été notées la veille de leur abattage de façon indépendante par les trois experts bouchers. Ces femelles, issues du cheptel de la ferme expérimentale de Jalogny (Saône-et-Loire), provenaient de diverses origines et présentaient ainsi des âges, des poids et des développements permettant de préjuger d'une grande hétérogénéité au sein de la population étudiée. La grille a été testée sur une population constituée de 38 vaches âgées de 32 à 128 mois (moyenne : $74 \pm 25$ mois) et 18 génisses âgées de 29 à 51 mois (moyenne : $39 \pm 5$ mois). La conformation des carcasses de ces femelles a été notée en majorité R et U (43 et 14 carcasses respectivement), 3 carcasses ayant des conformations plus extrêmes ( 2 en $E$ et 1 en $O$ ). Le lendemain de l'abattage, le grain de viande a été noté sur la carcasse selon la grille établie dans l'étape précédente (Oury et al 2008, 2010a).

La grille a été construite autour de 16 indicateurs évalués dans les jours précédant l'abattage et regroupés en deux grands domaines : d'une part les caractéristiques de l'animal et son développement, d'autre part les caractères raciaux et les aptitudes fonctionnelles (tableau 2). Le domaine « caractéristiques de l'animal et développement » comporte 9 indicateurs notés entre 1 et 5 (notamment relatifs à l'épaisseur de flanchet, l'équilibre avant-arrière ou encore la musculature). Pour chaque indicateur, la note de 5 a été attribuée à la modalité la plus favorable au grain de viande selon les professionnels. Le domaine « caractères raciaux et aptitudes fonctionnelles » comporte 7 indicateurs également notés entre 1 et 5 (par exemple le port des cornes, l'étranglement et l'aspect général de la tête ou encore la forme du museau). L'évaluation des 16 indicateurs faisant appel aux différents sens, notamment à la vue et au toucher, les maniements et lieux d'observation ont été consignés dans une planche de photos complétant la grille de notation.

Parmi les 16 indicateurs évalués sur l'animal vivant, 11 se sont révélés très concordant avec l'appréciation globale du grain de viande sur la carcasse (CCK $>0,57 ; \mathrm{P}<0,0001)$. Le niveau de concordance entre le grain de viande et les indicateurs en vif demeure cependant inférieur à celui existant entre le grain de viande et les postes d'évaluation sur la carcasse (Oury et al 2008, 2010a).

Parmi les indicateurs évalués en vif, certains ne sont pas reliés au grain de viande évalué sur la carcasse, notamment la docilité, l'aspect général de la tête, les caractéristiques de couleur, de taille et d'orientation des cornes.

Certains indicateurs ont une évolution synchrone par rapport à celle du grain de viande : l'épaisseur du cuir et son élasticité, la largeur du dos et la présence d'aloyau, la musculature de l'avant et celle de l'arrière $(0,71<\mathrm{CCK}<0,79$; $\mathrm{P}<0,001)$. Enfin, d'autres indicateurs ont permis de distinguer uniquement les 
Tableau 2. Indicateurs retenus pour le jugement du grain de viande sur animal en vif.

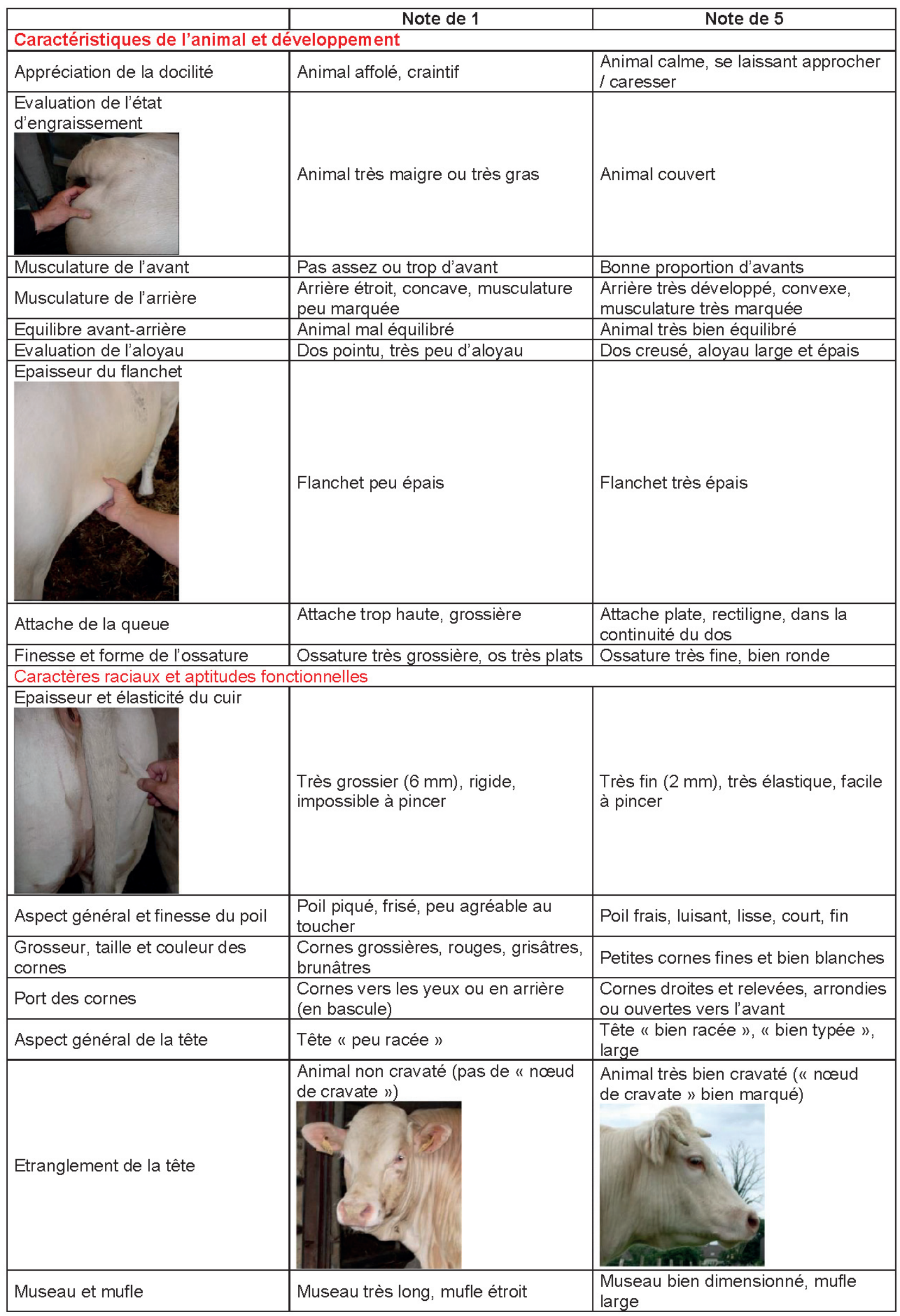


Figure 4. Note moyenne de différents indicateurs observés sur l'animal en vif (épaisseur de cuir et finesse de l'ossature), selon la note de grain de viande de la carcasse (résultats obtenus sur 60 femelles Charolaises évaluées en vif puis après abattage) (d'après Oury et al 2008, Ellies-Oury et al 2011).

Note moyenne d'épaisseur de cuir

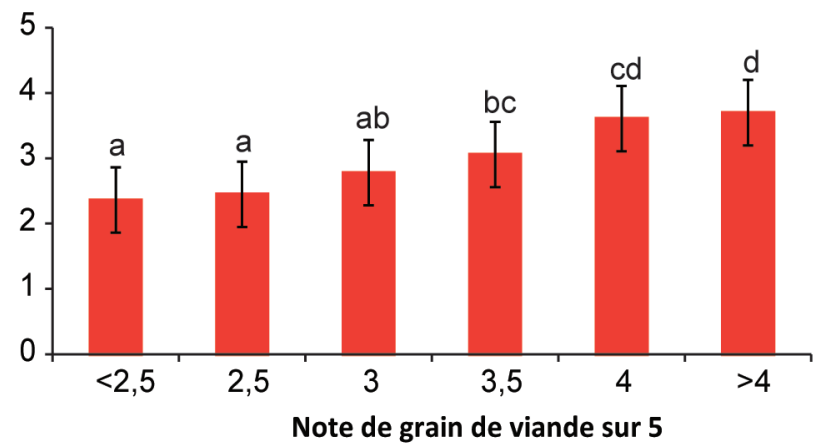

Note moyenne de finesse de l'ossature

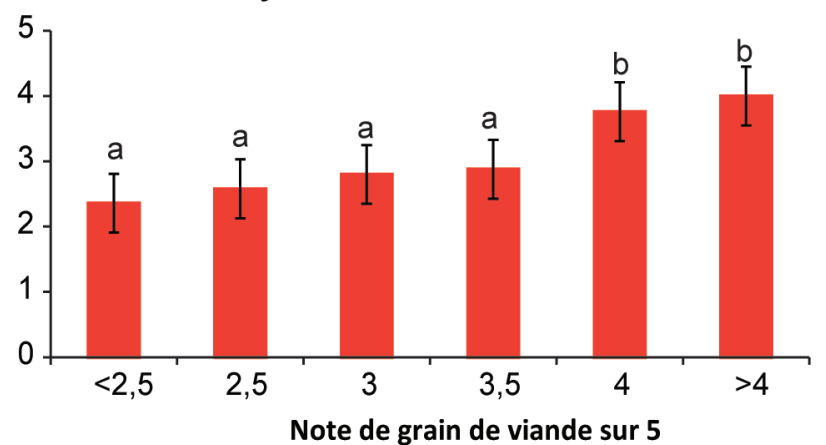

Une note de 5 pour chacun de ces descripteurs implique : un cuir très fin $(2 \mathrm{~mm})$, très élastique, facile à pincer ; une ossature très fine et bien ronde. Des lettres différentes témoignent d'une différence significative $(P<0,05)$.

grains fins (notés 4 ou plus) : l'équilibre avant arrière, l'épaisseur de flanchet, la finesse de l'os (figure 4).

Il a donc été mis en évidence que « l'on a plus de chance »d'avoir une viande à grain extrêmement fin, en choisissant un animal bien équilibré (avant/arrière), « avec de l'aloyau », une bonne proportion d'avants et un arrière très développé, un cuir très fin, très élastique et facile à pincer, une ossature fine et un flanchet très épais. Néanmoins, de moins en moins de bouchers achètent sur pied un animal et il semble donc que l'utilisation de cette grille de notation sur animal vif restera limitée. La grille de notation sur carcasse est en effet mieux adaptée pour répondre aux attentes des bouchers et de la filière. Aussi, la notation des indicateurs sur la carcasse entière et sur les quartiers après découpe est plus à même d'augmenter significativement les chances d'avoir un grain fin par rapport à la seule notation de l'animal vivant.

Suite à l'objectivation de ce savoirfaire, des points restaient à valider, notamment sur les propriétés physicochimiques des muscles et sensorielles des viandes selon qu'ils sont issus de carcasses à grains viande fin (note $>4$ ) ou grossier $(\text { note }<2)^{2}$. Deux études ont donc été réalisées dans cet objectif.

\section{3 / Caractérisation physico- chimique et sensorielle des muscles de carcasses ayant des notes de grain de viande extrêmes}

Au plan des savoir-faire, l'étude ethnologique a permis de conclure que cette notion était positive et était fré- quemment utilisée sur le terrain pour sélectionner et orienter les carcasses en fonction de leur potentiel de tendreté, tout du moins en zone Charolaise. Au plan de la physiologie musculaire, on peut admettre que le grain de viande correspond au réseau observable sur une coupe transversale de muscle, à différentes échelles microscopiques et macroscopiques. Il intègre à la fois le diamètre des fibres musculaires, la matrice extracellulaire qui les entoure et les lipides qu'elle contient (Purslow 2005, Albrecht et al 2006). Le grain de viande est ainsi identifiable visuellement et au toucher (Lepetit et Culioli 1994, Taylor 1998).

\section{1 / Le lien entre grain de viande et caractéristiques physicochimi- ques est dépendant du muscle considéré}

Suite à la formalisation de la notion de grain de viande, nous avons cherché à caractériser les principaux muscles utilisés par les experts : les muscles rectus abdominis (RA - bavette de flanchet ; évaluation dans la grille en vif), Longissimus Thoracis (LT - faux-filet ; prélevé au niveau de la $6^{\text {eme }}$ côte ; évaluation sur l'ART8), longus colli (LC - chaînette ou filet de côte ; évaluation sur la carcasse entière) et diaphragma (D - hampe ; évaluation sur la carcasse entière). Ces quatre muscles ont été prélevés 24 heures post-mortem sur 10 génisses Charolaises de même âge (35 mois), même poids $(405 \mathrm{~kg})$ et même état d'engraissement ( 3 sur 5). Ces 10 carcasses ont été sélectionnées selon leurs grains de viande extrêmes ( 5 grains fins et 5 grains grossiers) afin d'analyser leurs caractéristiques physicochimiques : force de cisaillement (Salé 1971), teneur et solubilité du collagène (Bergman-Loxley
1963, Bonnet et Kopp 1992) et teneur en lipides (NF V 04-402, 1968), activités enzymatiques (Ansay 1974, Briand et al 1981, Piot et al 1998) de leurs muscles (Ellies-Oury et al 2011). Certaines propriétés n'ont cependant pas pu être étudiées pour l'ensemble des muscles en raison de la taille limitée de ceux-ci. Ainsi, les mesures de force de cisaillement n'ont concerné que les muscles rectus abdominis et diaphragma et seules les activités enzymatiques ont pu être évaluées sur le muscle longus colli.

Sur la viande crue, lorsque les muscles rectus abdominis et diaphragma sont analysés ensemble, les forces de cisaillement sont significativement plus faibles pour les animaux à grain fin (tableau 3 ). Sur la viande cuite, pour le muscle rectus abdominis des animaux à grain fin, les forces de cisaillement sont également moindres que celles des viandes à grain grossier $(5,89 v s 7,20 \mathrm{~kg})$. Pour le muscle diaphragma, les écarts ne sont pas significatifs. La force plus faible mesurée sur le muscle rectus abdominis cuit pourrait trouver une explication dans des teneurs plus faibles et une solubilité supérieure du collagène, même si, dans ce cas, les écarts ne sont pas significatifs en raison d'une trop forte variabilité intra-lot. Cette hypothèse est confortée par la teneur en collagène significativement plus faible des muscles diaphragma et longissimus thoracis des animaux à grain fin. La moindre teneur en collagène du muscle longissimus thoracis est cohérente avec l'évaluation des experts qui ont jugé les nerfs peu présents sur le longissimus thoracis lors de l'évaluation des carcasses.

Il est donc possible de considérer que les experts sont effectivement capables, à travers la notation du grain de viande,

\footnotetext{
2 Ont été considérées comme « grain fin » et « grain grossier » des carcasses ayant un note de grain de viande supérieure à 4 et inférieure à 2 respectivement, cette notation étant attribuée par les quatre experts sollicités pour la validation de la grille de notation sur carcasse.
} 
Tableau 3. Propriétés physico-chimiques des 4 muscles étudiés selon le grain de viande de la carcasse (d'après Oury et al 2010a).

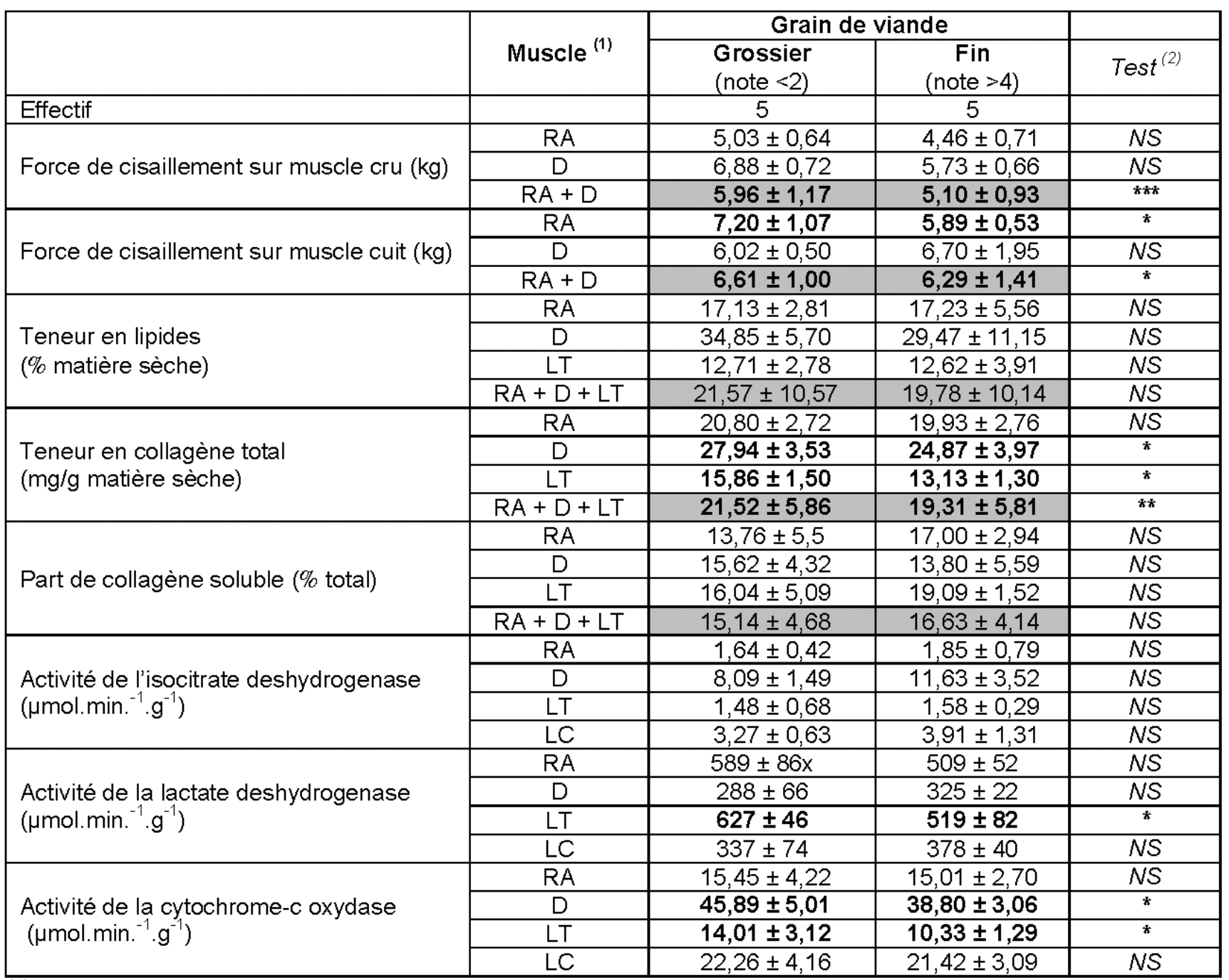

(1) RA : Rectus Abdominis ; D : Diaphragma ; LT : Longissimus Thoracis ; LC : Longus Colli.

(2) Moyenne \pm Ecart-type ; *** : $P<0,001 ; * *: P<0,01 ;{ }^{*}: P<0,05 ; N S: P>0,10$.

Deux types de tests ont été réalisés : 1) des ANOVA à un facteur testant l'effet du groupe de grain de viande sur le paramètres physicochimiques pour un muscle donné ainsi que 2) des ANOVA à un facteur avec répétitions (les muscles) testant l'effet du groupe de grain de viande sur les paramètres physicochimiques tous muscles confondus.

de noter certaines caractéristiques physicochimiques reliées à la tendreté/dureté de la viande (Delavigne 2008a et b, Oury et al 2008, 2010a). Les muscles diaphragma et longissimus thoracis du lot d'animaux à grain fin ont des activités oxydatives inférieures (cytochrome-c oxydase), les muscles LT présentant également une activité glycolytique inférieure (lactate déshydrogénase). Aucun écart significatif n'est en revanche noté au niveau de l'activité de l'enzyme oxydative isocitrate déshydrogénase, illustrant une réactivité des enzymes variable selon leur localisation (chaîne respiratoire, cycle de Krebs).

Dans cet essai, peu de mesures ont pu être réalisées sur le muscle longus colli (chaînette ou filet de côte) car c'est un muscle qui est plus délicat à échantillonner que les autres muscles étudiés ici.
Même s'il est souvent mis en avant par les professionnels, il ne peut être un muscle référence au laboratoire.

\section{2 / Quelques liens entre le} grain de viande et la qualité sensorielle sur le muscle longissimus thoracis

Dans un dernier temps, nous avons cherché à évaluer le bien-fondé de la notion de grain de viande pour prédire la qualité sensorielle des viandes et notamment la tendreté (Ellies-Oury et al 2011, $2013 \mathrm{a}$ et b). Les muscles longissimus thoracis prélevés au niveau de la $6^{\text {ème }}$ côte de 30 femelles extrêmes en termes de grain de viande (15 grains fins et 15 grains grossiers évalués selon la grille précédemment développée) ont été prélevés 24 heures post mortem puis maturés pendant 14 jours. Comme précédemment, les animaux ont été comparés à même âge (43 mois) et même poids de carcasse $(385 \mathrm{~kg})$. L'évaluation sensorielle a été réalisée par 12 jurés entraînés sur des steaks saignants (cuits à $55^{\circ} \mathrm{C}$ à cœur). Quelques paramètres physicochimiques classiques ont également été déterminés en parallèle (force de cisaillement, teneur en collagène et en lipides) selon les techniques détaillées en 3.1 .

Les teneurs en lipides des muscles des animaux à grain fin se sont révélées significativement plus faibles $(9,6$ vs $16,1 \%$ $\mathrm{MS} ; \mathrm{P}=0,005)$ que celles des muscles des animaux à grain grossier. Ces écarts de teneurs en lipides ont conduit à des notes d'intensité de flaveur plus faibles $(\mathrm{P}=0,09)$ pour les muscles du lot « grain fin ». La même tendance est relevée sur les notes de jutosité sans que l'écart ne soit significatif $(\mathrm{P}=0,78)$ (tableau 4$)$. 
Tableau 4. Propriétés physicochimiques et sensorielles du muscle longissimus thoracis selon le grain de viande de la carcasse (d'après Ellies-Oury et al 2011, 2013a et b).

\begin{tabular}{|l|c|c|c|}
\hline $\begin{array}{l}\text { Caractérisation du grain de viande } \\
\text { de la carcasse }\end{array}$ & $\begin{array}{c}\text { Grain Grossier } \\
(\text { note }<2)\end{array}$ & $\begin{array}{c}\text { Grain Fin } \\
(\text { note }>4)\end{array}$ & Test $^{(1)}$ \\
\hline Effectif & 15 & 16 & \\
\hline Teneur en lipides (\% matière sèche) & $\mathbf{1 6 , 1 \pm \mathbf { 5 , 7 }}$ & $\mathbf{9 , 6 \pm 3 , 8}$ & $* *$ \\
\hline Force de cisaillement viande crue (daN) & $3,0 \pm 0,6$ & $2,7 \pm 0,6$ & $\mathrm{NS}$ \\
\hline $\begin{array}{l}\text { Teneur en collagène total } \\
\text { (mg / g matière sèche) }\end{array}$ & $14,5 \pm 1,9$ & $15,6 \pm 3,1$ & $\mathrm{NS}$ \\
\hline Collagène soluble (\% total) & $17,7 \pm 4,1$ & $19,8 \pm 7,5$ & $\mathrm{NS}$ \\
\hline Tendreté initiale sur 10 & $7,4 \pm 0,6$ & $7,2 \pm 0,9$ & $\mathrm{NS}$ \\
\hline Tendreté globale sur 10 & $7,0 \pm 0,8$ & $6,8 \pm 0,9$ & $\mathrm{NS}$ \\
\hline Jutosité sur 10 & $6,1 \pm 0,8$ & $6,0 \pm 1,0$ & $\mathrm{NS}$ \\
\hline Intensité de flaveur sur 10 & $\mathbf{6 , 2} \mathbf{0 , 6}$ & $\mathbf{5 , 6 \pm 0 , 6}$ & $\mathbf{+}$ \\
\hline
\end{tabular}

${ }^{(1)}$ Moyenne \pm Ecart-type ; ${ }^{* *}: \mathrm{P}<0,01 ;+: 0,05<\mathrm{P}<0,10 ; N S: \mathrm{P}>0,10$.

Contrairement aux attentes, il n'est pas apparu de différence significative de tendreté initiale/globale entre les muscles des deux lots $(\mathrm{P}=0,58$ et $\mathrm{P}=0,50$ respectivement). Il est possible que les teneurs plus élevées en lipides intramusculaires des muscles des animaux à grain grossier aient influencé la perception de la tendreté en bouche par les dégustateurs. Néanmoins, il faut noter que les résultats des analyses sensorielles sont corroborés par l'absence de différence significative de force de cisaillement $(P=0,33)$, de teneur en collagène et de solubilité du collagène $(\mathrm{P}=0,23$ et $\mathrm{P}=0,33$ respectivement) entre les deux lots de grain de viande. Il n'est donc pas possible de tester le caractère prédictif des teneurs en collagène du muscle longissimus thoracis pour la notation $\mathrm{du}$ grain de viande, précédemment mis en évidence. Il est possible de faire l'hypothèse que le muscle longissimus thoracis n'est pas le meilleur muscle pour prédire le grain de viande. En effet, selon les experts, ce muscle est presque toujours tendre et permet donc difficilement de démasquer, à lui seul, des variations de tendreté entre animaux. Au vu de ces conclusions, il pourrait être intéressant de tester la variabilité des propriétés sensorielles et notamment de la tendreté des muscles rectus abdominis et/ou diaphragma selon que les carcasses sont issues de lots à grain fin ou grossier.

\section{Conclusion}

Ces études ont permis dans un premier temps la formalisation d'une notion empirique et d'un ressenti des différents intervenants de la filière viande bovine en race Charolaise. L'outil formalisé que représente la grille de notation est utilisé pour qualifier les carcasses de façon moins subjective qu'auparavant et orienter certains cahiers des charges.

Les quatre muscles indicateurs étudiés présentent bien des propriétés physicochimiques différentes selon que le grain est fin ou grossier. Il s'agit en particulier de la force de cisaillement, de la teneur en collagène et de l'activité de la cytochrome-c oxydase.
Contrairement aux attentes, les propriétés sensorielles (et notamment la tendreté) des muscles longissimus thoracis issus de carcasses à grain fin ou grossier ne sont pas ressorties significativement différentes. Néanmoins, l'écart de teneurs en lipides intramusculaires était en faveur des animaux à grain grossier, ce qui est susceptible d'avoir induit un biais dans les résultats. Aussi, les résultats de ces différentes études mériteraient-ils d'être confirmés à même teneur en lipides, sur une plus grande population, mais aussi sur des carcasses plus homogènes en termes de conformation et d'état d'engraissement.

En fonction de ces résultats, il pourrait également être envisagé de poursuivre les travaux sur l'impact des facteurs biotechniques ou des pratiques d'élevage sur le grain de viande afin de conseiller éventuellement les éleveurs sur leurs pratiques.

\section{Remerciements}

La première partie de ce travail a été réalisé avec le soutien financier de l'ANR - Agence Nationale de la Recherche dans le cadre du programme «Agriculture et Développement Durable », projet « ANR-05-PADD-012, Promotion du Développement Durable par les Indications Géographiques PRODDIG », d'AgroSupDijon et du Conseil Régional de Bourgogne. La seconde partie de cette étude a bénéficié du soutien du Conseil Régional de Bourgogne. Les auteurs remercient l'ensemble des professionnels de la filière pour l'intérêt qu'ils ont porté à cette recherche, en particulier C. Ducoté, D. Liodenot, J.L. Nelly et R. Sandrin. Les auteurs remercient F. Delamarche, J. Lambert, M. Jouanno pour les analyses physicochimiques et sensorielles.

\section{Références}

Albrecht E., Teuscher F., Ender K., Wegner J., 2006. Growth- and breed-related changes of marbling characteristics in cattle. J. Anim. Sci., 84, 2959-2964.

Ansay M., Laurent E., Roupain J., 1974. Individualité musculaire chez le bovin : étude de l'équipement enzymatique de quelques muscles. Ann. Biol. Anim. Bioch. Biophys., 14, 471-486.

Bergman I., Loxley R., 1963. Two improved and simplified methods for the spectrophotometric determination of hydroxyproline. Anal. Chem., 35, 1961-1965.

Bonnet M., Kopp J., 1992. Préparation des échantillons pour le dosage et la caractérisation qualitative du collagène musculaire. Viandes Prod. Carnés, 13, 87-91.
Briand M., Tamant A., Briand Y., Monin G., Durand B., 1981. Metabolic types of muscle in the sheep: I myosin ATPase glycolytic and mitochondrial enzyme activities. Eur. J. Appl. Physiol., 46, 347-358.

Delavigne A.E., 2008a. Usage de la notion de "grain de viande ". A propos d'un savoir faire propre aux professionnels de la viande. Ethno-zoo., 81, 67-77.

Delavigne A.E., 2008b. Le savoir-faire des bouchers et des chevillards en matière de choix des animaux et des carcasses. 12 $2^{\text {èmes }}$ Journées Sciences du Muscle et Technologie de la Viande. Viandes Prod. Carnés, Hors-Série, 26, 29-30.

Ellies-Oury M.P., Durand Y., Picard B., Micol D., Dumont R., 2011. Formalisation et validation de la notion de « grain de viande » comme prédicteur de la tendreté de la viande chez le bovin Charolais. Renc. Rech. Rum., 177-180.

Ellies-Oury M.P., Durand Y., Delamarche F., Jouanno M., Lambert J., Micol D., Dumont R., 2013a. Relationships between expert estimate of "grain of meat" and meat tenderness on Charolais cattle. Meat Sci., 93, 397-404.

Ellies-Oury M.P., Durand Y., Micol D., Dumont R., 2013b. Evaluation précoce de la qualité des viandes bovines - Relations entre l'évaluation du grain de viande sur les carcasses et la qualité sensorielle des viandes chez les bovins Charolais. Viandes Prod. Carnés, 8 p.

Grenet C., Mourier C., Geay Y., Jurie C., Picard B., Colin G., Durand Y., Le Pichon D., 2000. Qualité de la viande. L'illusoire tri 
physiologique des génisses. Viandes Prod. Carnés, 21, 173-179.

Jabet S., Turin F., 1997. Etude des possibilités de mise au point d'une méthode d'appréciation fiable du " grain de viande ». Institut de l'Elevage. Technopole Viandes du Limousin, $11 \mathrm{p}$.

Lepetit J., Culioli J., 1994. Mechanical properties of meat. Meat Science, 36, 203-237.

NF 04-402, 1968. Viandes et produits à base de viande - détermination de la teneur en matière grasse totale.

Oury M.P., Dumont R., Durand Y., 2008. Construction d'une grille de notation du grain de viande chez les bovins Charolais. $12^{\text {èmes }}$ JSMTV, Tours, France, 151-152.

Oury M. P., Durand Y., Micol D., Dumont R., 2010a. Construction et validation d'une grille de notation du " grain de viande » sur la carcasse à partir des savoir-faire des professionnels de la filière. Viandes Prod. Carnés, 28, 35-40.

Oury M.P., Quillard F., Durand Y., Micol D., Dumont R., 2010b. Construction d'une grille de notation du " grain de viande » sur animal vivant chez les bovins charolais. $13^{\text {èmes }}$ JSMTV, Clermont-Ferrand, France, 213-214.

Piot C., Veerkamp J.H., Bauchart D., Hocquette J.F., 1998. Contribution of mitochondria and peroxisomes to plamitate oxidation in rat and bovine tissues. Comp. Biochem. Phys., 121, 69-78.

Purslow P.P., 2005. Intramuscular connective tissue and its role in meat quality. Meat Sci., $70,435-447$

Salé P., 1971. Evolution de quelques propriétés mécaniques du muscle pendant la maturation. Bull. Tech. C.R.Z.V., Theix, 6, 35-44.

Taylor R., 1998. Structural basis for meat toughness and tenderness. Pol. J. Food Nutr. Sci., 7, 37-52.

\title{
Résumé
}

L'évaluation du « grain de viande » est une appréciation subjective, utilisée par certains chevillards et bouchers pour sélectionner et orienter les carcasses, un grain extrêmement fin étant synonyme de qualité. Pour évaluer la pertinence de cette notion quatre études ont permis $i$ ) d'objectiver la notion de grain de viande par une approche ethnologique, ii) de formaliser ce savoir-faire dans une grille de notation en vif et en carcasse, iii) d'évaluer les propriétés physicochimiques des quatre muscles utilisés par les experts pour juger une carcasse, et $i v$ ) d'évaluer l'existence d'un lien entre l'évaluation du grain de viande et la qualité sensorielle des viandes. Les seize indicateurs consignés dans la grille établie sur la carcasse sont répétables et facilement utilisables en abattoir. Sur le muscle longissimus thoracis, les grains fins ont été caractérisés par des teneurs en lipides intramusculaires significativement plus faibles que les grains grossiers, conduisant à des notes d'intensité de flaveur également significativement plus faibles, mais sans répercussions sur les notes de jutosité. Il n'est pas apparu de différence significative de tendreté entre les muscles à grains fins et grossiers. Au niveau physicochimique, le grain fin a été associé à des muscles nécessitant une moindre force de cisaillement sur la viande cuite et présentant une moindre teneur en collagène, sans différence sur sa solubilité, laissant supposer un effet favorable sur les propriétés sensorielles. Les écarts sont cependant très dépendants du muscle étudié et méritent d'être confirmés à plus grande échelle.

\begin{abstract}
Objectivation of the "grain of meat" notion and prospect of utilisation to evaluate the tenderness of Charolais beef meat

The assessment of the meat "grain" size is an empirical method used by some butchers in France to predict the tenderness of meat: the finer the grain the higher the quality. Four studies are presented here. After a characterization of this notion using an ethnological approach, two grids were built to objectivate the way of evaluating grain of meat on live animals and carcasses. The second aim was to characterize the main muscles used by the experts to rate the grain of meat. The last aim was to establish the relation between the score of meat grain estimated on the carcass and the tenderness of the longissimus thoracis muscles estimated by sensory evaluations. A group of 16 criteria were identified on the carcass as effective to anticipate overall "grain of meat" score. "Fine grain" carcasses were associated with lower lipid content than "coarse grain" in longissimus thoracis muscles, leading to significantly lower scores of flavor intensity without any impact on juiciness scores. No significant relation appeared between grain of meat and tenderness. The shear force needed to cut a broiled sample was found significantly higher for "coarse grain" carcasses than for "fine grain" ones. The total collagen in the "coarse grain" group was significantly higher than those from the "fine grain" group without any impact on collagen solubility. Taken together, these results allow hypothesizing that "fine grain" could have a positive impact on meat quality traits. The differences underlined are nevertheless very muscle-dependant and remain to be confirmed in larger trials.
\end{abstract}

ELLIES-OURY M.-P., DURAND Y., DELAVIGNE A.-E., PICARD B., MICOL D., DUMONT R., 2014. Objectivation de la notion de grain de viande et perspectives d'utilisation pour évaluer la tendreté des viandes de bovins Charolais. INRA Prod. Anim. 27, 347-358. 